\title{
Influence of Pre and Post-emergence Herbicides on Weed Dynamics, Growth and Yield of Soybean (Glycine max L.)
}

\author{
Gabu Singh Gathiye ${ }^{1 *}$,Subhash Bhanwar ${ }^{2}$ and Vishal Verma ${ }^{3}$ \\ ${ }^{1}$ RVSKVV-Krishi Vigyan Kendra, Dhar (M.P.), India \\ ${ }^{2}$ Dr. B.R. Ambedkar University of Social Sciences, Mhow (M.P.), India \\ ${ }^{3}$ Tropical Forest Research Institute, Jabalpur (M.P.), India
}

*Corresponding author

\section{Keywords}

Pre-emergence herbicides, Postemergence herbicides, Weed species, Weed index, Weeds control efficiency (WCE) and yield

\section{Article Info}

Accepted:

26 April 2020

Available Online:

10 May 2020

\author{
A B S T R A C T
}

An experiment entitled "Influence of pre and post-emergence herbicides on weed dynamics, growth and yield of soybean (Glycine max L.)" was conducted in Kharif season 2015 at research farm of BRAUSS, MHOW, (M.P.). The soil of the experimental field was medium black in texture, neutral in reaction $(\mathrm{pH} 7.60)$ with normal EC $(0.45 \mathrm{dS} / \mathrm{m})$ and medium organic carbon contents $(0.72 \%)$ and analysing low in available $\mathrm{N}(270 \mathrm{~kg} / \mathrm{ha})$, medium in available $\mathrm{P}(6.9 \mathrm{~kg} / \mathrm{ha})$ and high in available $\mathrm{K}(382 \mathrm{~kg} / \mathrm{ha})$ contents. Due to dominance of montmorillonite clay content it has high capacity to swell and shrink and high CEC. A field experiment was consisted of 9 treatments replicated four times in randomized block design (RBD). As per treatment, the seed of soybean $c v$. JS 335 was sown in all the treatments consisting with pre and post emergence herbicides. The treatment $\mathrm{T}_{4}$ - Fenoxaprop-p-ethyl $9 \%$ EC, $67.5 \mathrm{~g} /$ ha post eme. (20 DAS) showed weed index or minimum decline $(2.3 \%)$ in seed yield. Among other treatments minimum decline $(4.95 \%)$ was recorded under treatment $\mathrm{T}_{5^{-}}$Chlorimuron ethyl + Fenoxaprop-ethyl, $9.37 \mathrm{~g} / \mathrm{ha}+67.5 \mathrm{~g} / \mathrm{ha}$, post emergence while highest yield decline $(36.22 \%)$ was observed under control $\left(\mathrm{T}_{8}\right)$. Weed control efficiency (WCE) was relatively higher in $\mathrm{T}_{9}$-weed free plots $(98.25 \%), \mathrm{T}_{4}$-fenoxaprop-p-ethyl $(97.98 \%)$ and $\mathrm{T}_{5}$-Chlorimufon-ethyl + fenoxaprop-p-ethyl $(97.84 \%)$ than rest of the treatments. The highest grain $(1.43 \mathrm{t} / \mathrm{ha})$ and stover yield $(1.55 \mathrm{t} / \mathrm{ha})$ was recorded under treatment $\mathrm{T}_{9}$-weed free plot followed by 1.4 t/ha and $1.47 \mathrm{t} /$ ha under treatment $\mathrm{T}_{4}$-fenoxaprop-p-ethyl $9 \% \mathrm{EC}$ while the lowest grain (0.91 t/ha) and straw yield (1.14 t/ha) was obtained under treatment $\mathrm{T}_{8}$ (control).

\section{Introduction}

Soybean (Glycine $\max$ (L) Merrill) is established as one of the prime monsoon season field crops in Madhya Pradesh particularly in Malwa plateau. It has resulted economical crop because of comparatively good economic return/unit area obtained by the farmers from its improvement in the living condition of farmers. Indian soybean holds on an average $37-41 \%$ protein, $17-21 \%$ oil, $25-$ $30 \%$ carbohydrate, $4-5 \%$ ash, $4-5 \%$ crude fiber and $2 \%$ phospholipids, Hence, it is called 'meat of the field'. However, its 
productivity in the State id $1102 \mathrm{~kg} / \mathrm{ha}$ which is very low as compared to the global productivity of $2206 \mathrm{~kg} / \mathrm{ha}$ (Anonymous, 2014)

Weeds are the major biotic factor responsible for poor yield in soybean. Simultaneous emergence and rapid growth of large number of weed species causes severe crop-weed competitions and reduction in crop yields (30$80 \%$ ) depending upon the type of weed flora and weed density. The incessant rains do not permit timely inter cultivations and manual control of weeds is also difficult on large scale on account of high cost and labour shortage during weeding peaks.

Therefore, there is a need for alternative methods of reducing the weed load during early crop growth period of soybean i.e. first 30-45 DAS. The herbicides presently available are either pre-emergence (PRE) or pre-plant incorporated (PPI) and have a narrow spectrum weed control. The biology of some weeds that occur in soybean makes it difficult to achieve effective weed control with single application of herbicides; PPI or PRE or post emergence (POST). Recent studies clearly indicated that combination application of herbicides (PRE followed by (fb) (POST) will provide more consistent weed control than single application. A well planned PRE fb POST herbicide application would provide more consistent weed control and helps to minimize the weed menace. Hence, present investigation was undertaken to study the effect of sequential application of pre and post-emergence herbicides in soybean. This study was undertaken to obtain an efficient herbicides weed control system and also to compare the relative efficacy of different herbicides with farmer's practice.

\section{Materials and Methods}

The experiment entitled "Influence of pre and post-emergence herbicides on weed dynamics, growth and yield of soybean (Glycine max L.)" was conducted on the Research Farm of BRAUSS Mhow in Rehati Hoshangabad, (M.P.). The topography of the experimental area are fairly leveled and proper drainage was provided. The plots were protected as not to allow the free flowing of surface runoff water, affecting the individual plot treatments. Hoshangabad is situated in Malwa plateau in Western parts of Madhya Pradesh on $22^{\circ} 43^{\prime} \mathrm{N}$ latitude and $75^{\circ} 66^{\prime} \mathrm{E}$ longitude an altitude of 555.5 metre above the mean sea level. This region enjoys subtropical semi arid type climate with an average rainfall of $940 \mathrm{~mm}$, most of which received during mid June to mid. September.

The soil of the experimental field has been grouped under medium black (Vertisols) belonging to fine montmorillonite hyperthermic family predominantly clay textural class. For fertility status of the experimental area, the soil samples were collected randomly with the help of soil augar before sowing from the experimental field and representative composite sample was made for the mechanical and chemical analysis. The soil of the experimental field was medium black in texture, neutral in reaction $(\mathrm{pH}$ 7.60) with normal EC (0.45 $\mathrm{dS} / \mathrm{m}$ ) and medium organic carbon contents $(0.72 \%)$ and analysing low in available $\mathrm{N}$ $(270 \mathrm{~kg} / \mathrm{ha})$, medium in available $\mathrm{P}$ (6.9 $\mathrm{kg} / \mathrm{ha}$ ) and high in available $\mathrm{K}(382 \mathrm{~kg} / \mathrm{ha})$ contents.

The experiment consisting of nine treatments and four replications with randomized block design was laid out in the experiment. Alachlor, Pendimetholin are the herbicides, which were used as pre emergence. These were sprayed immediately after the sowing of soybean crop. Chlorimuron ethyl, fenoxoprop ethyl. Chlorimuron ethyl + fenoxoprop ethyl, quizaifop ethyl, and imazethapyr were used as post emergence herbicide. These were sprayed at 15-25 days after planting as per 
herbicide. The herbicides spray mixture was added with $1 \mathrm{ml}$ per litre of gum as stickers. The following pre and post harvest observations were studied during the experiment:

\section{Studies on weeds}

\section{Weed species}

Different weeds species presenting the experimental area during the crop season were identified. Effect of different treatments was assessed on the intensity and growth of the weeds at 15, 30, 45, 60 days of sowing and at maturity. The number of weeds in a quadrate of $50 \mathrm{~cm}$ at three random spots in each plot was counted.

\section{Weed index}

Weed index may be defined as the percent reduction in the yield due to presence of weeds in weeds in comparison to weed free condition (hand weeding) mathematically it could be expressed as below:

Weed index (W.I.) $=\mathrm{X}-\mathrm{Y} / \mathrm{X} \times 100$

Where,

$\mathrm{X}=$ Yield from weed free plot (hand weeding)

$Y=$ Yield from the treated plot for which weed index is to be worked out.

\section{Weed control efficiency (WCE)}

Weed control efficiency measures the efficiency of any weed control treatment in comparison to no weeding treatment. Mathematically, it could be expressed as below (Mani et al., 1973).

$\mathrm{WCE}=\quad \mathrm{DWC}-\mathrm{DWT} / \mathrm{DWC} \times 100$
Where,

$\mathrm{WCE}=$ Weed control efficiency

DWC $=$ Dry weight of weeds in treated plot

DWT $=$ Dry weight of weeds in untreated plot

\section{Studies on growth parameters}

\section{Plant population}

Initial and final plant population of crop of crop was counted at 20 days after sowing and just before harvesting respectively in one $\mathrm{m}$ row length at three random places in three different rows in each net plot and mean was worked out.

\section{Number of leaves per plant}

The number of leaves was counted on the 5tagged plants per plot and mean was calculated. These observations were taken at $30,45,60,75$ DAS in all the treatments.

\section{Number of root nodules per plant}

The total number of root nodules obtained from the 5 plants was counted and the average number of nodules per pant was thus computed. This observation was taken at 45 DAS.

\section{Studies on yield parameters}

\section{Seed yield per plant}

Average seed yield per plant was derived from the produce of the 10 sample plants those were drawn randomly from each of the net plots.

\section{Stover yield per plant}

The stover yield per plant was obtained by subtracting grain yield from randomly 
selected fine plants weight from each plot and averages were worked out.

\section{Biological yield (t/ha)}

Biological yield is the total yield of crop including economical yield and the stover yield. The biological yield per net plot was recorded after harvesting of the crop plants. The plot yield was later on converted into t/ha by multiplying it by conversion factor.

\section{Seed yield (t/ha)}

The seed yield per net plot was recorded after drying the seed it is also known as economical yield. The plot yield was later on converted in to t/ha by multiplying it by conversion factor.

\section{Stover yield (t/ha)}

The stover yield per plot was obtained by subtracting grain yield (economical yield) from biological yield (bundle weight) in each plot. This was later on converted in to $\mathrm{t} / \mathrm{ha}$.

\section{Results and Discussion}

\section{Studies on the weeds}

\section{Weed species}

Different weeds species presenting the experimental area during the crop season were identified and are listed as under (Table 1) in descending order of their density in the weedy field.

\section{Number of monocot weeds $/ \mathrm{m}^{2}$}

The population of monocot weeds revealed that application of pre-emergence herbicides lowered the population of monocot weeds to a great extent and was significantly superior to untreated plots. Pendimethalin was found to be superior to alachlor at this respect. At 30 DAS, the application of post emergence applied fenoxaprop-p-ethyl herbicide (20 DAS) gave significantly lower density of monocot weeds than that recorded in weedy check (Table 2).

Observations at 45 DAS showed that application of post-emergence herbicides killed majority of monocot weeds and the effect of fenoxaprop-ethyl was found to be relatively more phytotoxic than quizalpfopethyl and imazethapyr. Weed free $\left(\mathrm{T}_{9}\right)$ recorded the lowest population monocot weeds but nearly equal to that observed under the application of fenoxaprop-ethyl. The maximum weed population was recorded under control followed by Chlorimuron-ethyl and pendimethalin. At 60 DAS monocot weeds were the highest under control $\left(23.24 / \mathrm{m}^{2}\right)$ followed by Chiorimuron-ethyl and Alachlor (15.52 and $11.35 / \mathrm{m}^{2}$ ) whereas in other treatments it was quite lower. At harvest, the weed free treatments resulted in the least number of monocot weeds/ $\mathrm{m}^{2}$ and hence work significantly superior to all the treatments except $\mathrm{T}_{4}$.

\section{Number of dicot weeds/ $\mathbf{m}^{2}$}

The number of dicot weeds at all the stages of crop growth remained numerically higher than the monocots. At 15 DAS, number of dicot weeds/ $\mathrm{m}^{2}$ was recorded more of less uniform in the untreated plots, whereas, it was well controlled in the pendimethalin and alachlor (pre-emergence) treated plots. At 30 DAS, highest number of dicot weeds/ $\mathrm{m}^{2}$ was recorded under weedy plot $\left(19.1 / \mathrm{m}^{2}\right)$ followed by quzalofop-ethyl $\left(12.35 / \mathrm{m}^{2}\right)$ and the lowest number was recorded under weed free $(0.6$ $\mathrm{m}^{2}$ ). When the post-emergence herbicides were applied at $20 \mathrm{DAS}$, the population of dicot weeds remained very much under control at all the stages, ahead (Table 3). 
Number of dicots weeds at 45 DAS revealed that amongst the herbicide quizalofop-ethyl showed the highest dicot weeds $\left(16.7 \mathrm{~m}^{2}\right)$. The lowest number or didcot weeds/ $\mathrm{m}^{2}$ were recorded under weed free condition $\left(0.9 / \mathrm{m}^{2}\right)$. At 60 DAS, weeds free treatment gave minimum number of dicor weeds $/ \mathrm{m}^{2}$, while, it was maximum under control plot. At harvest the minimum number of dicots weeds/m2 was observed under Chorimuronethyl $\left(4.2 / \mathrm{m}^{2}\right)$ followed by Chlorimuron ethyl + fenoxaprop-p-ethyl $\left(5.1 / \mathrm{m}^{2}\right)$ after weed free plots. At this stage highest number of dicot weeds were recorded under control $\left(21.11 / \mathrm{m}^{2}\right)$ followed by quizalofop-p-ethyl $\left(13.3 / \mathrm{m}^{2}\right)$.

\section{Dry weight of weeds}

The weed free treatment $\left(\mathrm{T}_{9}\right)$ proved significantly superior to all other treatments of the experiment, the lowest dry weight of $1.90 \mathrm{~g} / \mathrm{m}^{2}$ was obtained at harvest followed by $\mathrm{T}_{4}$ and $\mathrm{T}_{5}$ under which dry weight of weeds was $2.20 \mathrm{~g} / \mathrm{m}^{2}$ (Fenoxaprop-p-ethyl), and 2.35 $\mathrm{g} / \mathrm{m}^{2}$ (Chlorimuron + ethyl fenoxaprop-pethly) respectively. Under the treatment $\mathrm{T}_{8}$ (control) maximum dry weight of $109.00 \mathrm{~g} / \mathrm{m}^{2}$ was obtained, thus Chlorimuron-ethyl $25 \%$ $\mathrm{WP}$ at 20 DAS and fenoxaprop-ethyl $9 \%$ EC In combination with Chlorimuron-ethyl $25 \%$ WP were more advantageous treatment as compared to all other treatments at harvest (Table 4).

At the crop stage of 60 DAS the treatment $\mathrm{T}_{9}$ (weed free plot) was significantly with dry weight of $1.60 \mathrm{~g} / \mathrm{m}^{2}$ as compared to 67.35 $\mathrm{g} / \mathrm{m}^{2}$ under $\mathrm{T}_{8}$ (control), while Chlorimuron ethyl $25 \%$ WP was recorded to have 6.70 $\mathrm{g} / \mathrm{m}^{2}$ and Chorimuron + fenoxaprop-ethyl combination treatment had $1.40 \mathrm{~g} / \mathrm{m}^{2}$, thus, the weedy check gave highest weed biomass $\left(109.0 \mathrm{~g} / \mathrm{m}^{2}\right)$ at harvest. All the chemical herbicide treatments recorded lower dry weight as compared to control $\left(\mathrm{T}_{8}\right)$ but significantly higher than weed free plot $\left(\mathrm{T}_{9}\right)$.

\section{Weed index}

Weed index i.e. yield reduction due to different treatments in comparison to control as presented in Table 5 revealed that among herbicide treatment $\mathrm{T}_{4}$ - Fenoxaprop-p-ethyl 9 $\%$ EC, $67.5 \mathrm{~g} / \mathrm{ha}$ post eme.(20 DAS) showed minimum decline $(2.3 \%)$ in seed yield. Among other treatments minimum decline $(4.95 \%)$ was recorded under treatment $\mathrm{T}_{5^{-}}$ Chlorimuron ethyl + Fenoxaprop-ethyl, 9.37 $\mathrm{g} / \mathrm{ha}+67.5 \mathrm{~g} / \mathrm{ha}$, post eme. Highest yield decline $(36.22 \%)$ was observed under control $\left(\mathrm{T}_{8}\right)$.

\section{Weed control efficiency (WCE)}

The WCE was relatively higher in $\mathrm{T}_{9}$-weed free plots (98.25\%), $\mathrm{T}_{4}$-fenoxaprop-p-ethyl $(97.98 \%)$ and $\mathrm{T}_{5}$-Chlorimufon-ethyl + fenoxaprop-p-ethyl $(97.84 \%)$ than rest of the treatments. It clearly brought out that all the herbicides except quizalofop-p-ethyl and imazethapyr gave reasonably higher WCE.

\section{Studies on growth parameters}

\section{Plant population (per m row length)}

Plant population per meter row length was recorded at 20 DAS and at harvest (Table 6). The data showed that plant population affected significantly by various weed management treatments, which comprised of different chemical herbicides, It was also noted that the plant population was fairly uniform and there was no mortality at any stage of crop growth till maturity of the crop.

\section{Number of leaves per plant}

Data revealed that treatment number $\mathrm{T}_{9}$ (weed free plot) proved significantly superior to all the treatments except $\mathrm{T}_{4}$ (Fenoxaprop-p-ethyl) at harvest stage. The minimum number of leaves at harvest was recorded in $T_{7}$ - 
Imazethapyr 5\% SL (14.60). The treatment $\mathrm{T}_{4}$ (fenoxaprop-p-ethyl) gave 20.20 number of leaves/plant at harvest, which was almost at par with $\mathrm{T}_{9}$-weed free plot (21.60).

Table.1 Weed species associated with soybean in experimental plots

\begin{tabular}{|c|c|c|c|c|}
\hline $\begin{array}{l}\text { S. } \\
\text { No. }\end{array}$ & $\begin{array}{l}\text { Hindi } \\
\text { name }\end{array}$ & $\begin{array}{l}\text { English } \\
\text { name }\end{array}$ & Botanical name & Family \\
\hline $\mathbf{A}$ & \multicolumn{4}{|c|}{ Monocotyledon weed species } \\
\hline 1 & $\begin{array}{l}\text { Janglli } \\
\text { Sawan }\end{array}$ & Wild rice & Echinochloa colonum & Gramineae \\
\hline 2 & $\begin{array}{l}\text { Janglli } \\
\text { Sawan }\end{array}$ & $\begin{array}{l}\text { Barnyard } \\
\text { grass }\end{array}$ & Echinochloa crusgalli & Gramineae \\
\hline 3 & Grass & - & Dinebra arebica & Poaceae \\
\hline 4 & Grass & Crab grass & Degitaria restroflexa & Poaceae \\
\hline 5 & Diwalia & Cynotis & Cynotis axillaria & Commelinaceae \\
\hline 6 & Bokhana & Day flower & Commelina benghalensis & Commelinaceae \\
\hline 7 & Motha & Prplenut sedge & Cyprus rotundus & Cyperaceae \\
\hline 8 & Doob & $\begin{array}{l}\text { Bermuda } \\
\text { Grass }\end{array}$ & Cyprus rotundus & Gramineae \\
\hline 9 & Chipkani & Foxtail & Setaria glauca & Poaceae \\
\hline B & \multicolumn{4}{|c|}{ Dicotyledonous weed species } \\
\hline 1 & Lehsua & Digera & Digera ervensis & Amaranthaceae \\
\hline 2 & Dudhi & Wild Poinsettia & Euphorbia geniculata & Euphorbiaceae \\
\hline 3 & Kuppi & Copper leaf & Acalypha indica & Euphorbiaceae \\
\hline 4 & Bhangra & Mukand & Eclipta alba & Asteraceae \\
\hline 5 & Gokharu & Cocklebur & Xanthium strumerium & Asteraceae \\
\hline 6 & - & Choti dudhi & E.microphylla & Euphorbiaceae \\
\hline 7 & Banmakoya & Ground cherey & Physalis minima & Solanaceae \\
\hline 8 & Jngli jute & Wild jute & Corchorus acutangulus & Tilliaceae \\
\hline 9 & - & - & Tridax procumbens & Asteraceae \\
\hline 10 & Badi dudhi & Badi dudhi & Euphorbia hirta & Euphorbiaceae \\
\hline 11 & $\begin{array}{l}\text { Chandni } \\
\text { Chatale }\end{array}$ & $\begin{array}{l}\text { Carrot grass of } \\
\text { Congress grass }\end{array}$ & Parthemium hysterophorus & Asteraceae \\
\hline 12 & & Prickly chafflower & Achyranthes aspera & Amaranthaceae \\
\hline
\end{tabular}


Table.2 Effect of different treatments on the monocot weeds at successive stage of growth

\begin{tabular}{|c|c|c|c|c|c|c|}
\hline \multirow{2}{*}{$\begin{array}{l}\text { Tr. } \\
\text { No. }\end{array}$} & \multirow[t]{2}{*}{ Treatments } & \multicolumn{5}{|c|}{ Number of monocot weeds } \\
\hline & & $\begin{array}{c}15 \\
\text { DAS }\end{array}$ & $\begin{array}{l}\text { 30 } \\
\text { DAS }\end{array}$ & $\begin{array}{c}45 \\
\text { DAS }\end{array}$ & $\begin{array}{c}\text { 60 } \\
\text { DAS }\end{array}$ & $\begin{array}{c}\text { At } \\
\text { Harvest }\end{array}$ \\
\hline $\mathbf{T}_{1}$ & Alachlor $50 \mathrm{EC}, 2.0 \mathrm{~kg} / \mathrm{ha}$ (Pre.eme.) & 4.1 & 6.50 & 8.9 & 9.2 & 6.2 \\
\hline $\mathbf{T}_{2}$ & Pendimethalin 30 EC, $750 \mathrm{~g} / \mathrm{ha}$ (Pre.eme.) & 4.7 & 8.60 & 10.25 & 11.5 & 7.15 \\
\hline $\mathbf{T}_{3}$ & $\begin{array}{l}\text { Chlorimuron-ethyl } 25 \% \text { WP, } 9.37 \mathrm{~g} / \mathrm{ha} \text {, post } \\
\text { eme. (20DAS) }\end{array}$ & 12.33 & 9.32 & 11.89 & 15.52 & 12.23 \\
\hline $\mathbf{T}_{4}$ & $\begin{array}{l}\text { Fenoxaprop-p-ethyl 9\% EC, } 67.5 \text { g/ha post eme. } \\
\text { (20DAS) }\end{array}$ & 11.70 & 2.10 & 1.7 & 1.02 & 0.95 \\
\hline $\mathbf{T}_{5}$ & $\begin{array}{l}\text { Chlorimuron ethyl + Fenoxaprop-ethyl, } 9.37 \mathrm{~g} / \mathrm{ha} \\
+67.5 \mathrm{~g} / \mathrm{ha} \text {, post eme. }\end{array}$ & 8.62 & 2.69 & 2.1 & 2.59 & 2.42 \\
\hline $\mathbf{T}_{6}$ & $\begin{array}{l}\text { Quizalafop-p-ethyl 5\% EC, } 50 \mathrm{~g} / \mathrm{ha} \text {, post eme. } \\
\text { (15 DAS) }\end{array}$ & 9.89 & 3.16 & 4.42 & 7.3 & 6.9 \\
\hline $\mathbf{T}_{7}$ & $\begin{array}{l}\text { Imazethapyr } 5 \% \mathrm{EC}, 75 \mathrm{~g} / \mathrm{ha} \text {, post eme. ( } 25 \\
\text { DAS) }\end{array}$ & 11.4 & 3.92 & 5.2 & 10.34 & 9.85 \\
\hline $\mathbf{T}_{8}$ & Control (unweeded) & 13.2 & 16.1 & 31.36 & 23.24 & 16.26 \\
\hline $\mathbf{T}_{9}$ & Weed free & 11.5 & 1 & 0.71 & 0.82 & 0.91 \\
\hline & Sem & 0.62 & 0.56 & 0.41 & 0.76 & 0.24 \\
\hline & $\mathrm{CD}$ at $5 \%$ & 1.81 & 1.66 & 1.21 & 2.23 & 0.72 \\
\hline
\end{tabular}

Table.3 Effect of different treatments on the dicot weeds at successive stage of plant growth

\begin{tabular}{|c|l|c|c|c|c|c|}
\hline $\begin{array}{l}\text { Tr. } \\
\text { No. }\end{array}$ & Treatments & \multicolumn{5}{|c|}{ Number of monocot weeds } \\
\cline { 4 - 7 } & & $\begin{array}{c}\mathbf{1 5} \\
\text { DAS }\end{array}$ & $\begin{array}{c}\mathbf{3 0} \\
\text { DAS }\end{array}$ & $\begin{array}{c}\mathbf{4 5} \\
\text { DAS }\end{array}$ & $\begin{array}{c}\mathbf{6 0} \\
\text { DAS }\end{array}$ & $\begin{array}{c}\text { At } \\
\text { Harvest }\end{array}$ \\
\hline $\mathbf{T}_{\mathbf{1}}$ & Alachlor 50 EC, 2.0 kg/ha (Pre.eme.) & 4.3 & 7.2 & 13.4 & 11.1 & 9.02 \\
\hline $\mathbf{T}_{\mathbf{2}}$ & Pendimethalin 30 EC, 750 g/ha (Pre.eme.) & 5.1 & 8.6 & 16.1 & 13.01 & 10.56 \\
\hline $\mathbf{T}_{\mathbf{3}}$ & $\begin{array}{l}\text { Chlorimuron-ethyl 25\% WP, 9.37 g/ha, post eme. } \\
\text { (20DAS) }\end{array}$ & 16.23 & 3.02 & 2.76 & 2.55 & 4.2 \\
\hline $\mathbf{T}_{\mathbf{4}}$ & $\begin{array}{l}\text { Fenoxaprop-p-ethyl 9\% EC, 67.5 g/ha post } \\
\text { eme.(20DAS) }\end{array}$ & 14.26 & 4.85 & 7.2 & 6.6 & 5.1 \\
\hline $\mathbf{T}_{\mathbf{5}}$ & $\begin{array}{l}\text { Chlorimuron ethyl + Fenoxaprop-ethyl, 9.37 g/ha } \\
\text { +67.5 g/ha, post eme. }\end{array}$ & 12.25 & 5.1 & 7.1 & 5.9 & 5.01 \\
\hline $\mathbf{T}_{\mathbf{6}}$ & $\begin{array}{l}\text { Quizalafop-p-ethyl 5\% EC, 50 g/ha, post eme. (15 } \\
\text { DAS) }\end{array}$ & 14.37 & 12.35 & 16.7 & 16.7 & 13.3 \\
\hline $\mathbf{T}_{\mathbf{7}}$ & Imazethapyr 5\% EC, 75 g/ha, post eme. (25 DAS) & 14.25 & 6.6 & 8.2 & 12.1 & 8.1 \\
\hline $\mathbf{T}_{\mathbf{8}}$ & Control (unweeded) & 15.01 & 19.1 & 28.3 & 32.1 & 21.11 \\
\hline $\mathbf{T}_{\mathbf{9}}$ & Weed free & 14.6 & 0.6 & 0.9 & 0.9 & 1 \\
\hline & Sem & 0.42 & 0.30 & 0.29 & 0.27 & 0.34 \\
\hline & CD at 5\% & 0.89 & 0.85 & 0.8 & 0.1 \\
\hline
\end{tabular}


Table.4 Dry weight of weeds $\left(\mathrm{g} / \mathrm{m}^{2}\right)$ at $30,45,60$ and 75 DAS and at harvest

\begin{tabular}{|c|c|c|c|c|c|c|}
\hline \multirow{2}{*}{$\begin{array}{l}\text { Tr. } \\
\text { No. }\end{array}$} & \multirow[t]{2}{*}{ Treatments } & \multicolumn{5}{|c|}{ Dry weight of weeds $\left(\mathrm{g} / \mathrm{m}^{2}\right)$} \\
\hline & & $\begin{array}{c}30 \\
\text { DAS }\end{array}$ & $\begin{array}{c}45 \\
\text { DAS }\end{array}$ & $\begin{array}{c}\text { 60 } \\
\text { DAS }\end{array}$ & $\begin{array}{c}75 \\
\text { DAS }\end{array}$ & $\begin{array}{c}\text { At } \\
\text { Harvest }\end{array}$ \\
\hline $\mathbf{T}_{1}$ & Alachlor $50 \mathrm{EC}, 2.0 \mathrm{~kg} / \mathrm{ha}$ (Pre.eme.) & 11.10 & 16.11 & 20.00 & 35.40 & 52.00 \\
\hline $\mathbf{T}_{2}$ & $\begin{array}{l}\text { Pendimethalin } 30 \mathrm{EC}, 750 \mathrm{~g} / \mathrm{ha} \\
\text { (Pre.eme.) }\end{array}$ & 9.40 & 10.00 & 22.25 & 29.60 & 48.10 \\
\hline $\mathbf{T}_{\mathbf{3}}$ & $\begin{array}{l}\text { Chlorimuron-ethyl 25\% WP, } 9.37 \\
\text { g/ha, post eme. (20DAS) }\end{array}$ & 4.10 & 2.10 & 6.70 & 8.80 & 27.10 \\
\hline $\mathbf{T}_{4}$ & $\begin{array}{l}\text { Fenoxaprop-p-ethyl } 9 \% \text { EC, } 67.5 \mathrm{~g} / \mathrm{ha} \\
\text { post eme.(20DAS) }\end{array}$ & 2.90 & 0.82 & 1.20 & 1.85 & 2.20 \\
\hline $\mathbf{T}_{5}$ & $\begin{array}{l}\text { Chlorimuron ethyl + Fenoxaprop- } \\
\text { ethyl, } 9.37 \mathrm{~g} / \mathrm{ha}+67.5 \mathrm{~g} / \mathrm{ha} \text {, post } \\
\text { eme. }\end{array}$ & 3.00 & 0.96 & 1.40 & 2.16 & 2.35 \\
\hline $\mathbf{T}_{6}$ & $\begin{array}{l}\text { Quizalafop-p-ethyl 5\% EC, } 50 \mathrm{~g} / \mathrm{ha} \text {, } \\
\text { post eme. (15 DAS) }\end{array}$ & 14.10 & 8.70 & 13.20 & 48.00 & 63.30 \\
\hline $\mathbf{T}_{7}$ & $\begin{array}{l}\text { Imazswthapyr } 5 \% \text { EC, } 75 \mathrm{~g} / \mathrm{ha} \text {, post } \\
\text { eme. (25 DAS) }\end{array}$ & 11.00 & 12.40 & 17.82 & 41.0 & 58.70 \\
\hline $\mathbf{T}_{8}$ & Control (unweeded) & 30.30 & 41.10 & 67.35 & 85.10 & 109.00 \\
\hline $\mathbf{T}_{9}$ & Weed free & 0.65 & 0.72 & 1.10 & 1.60 & 1.90 \\
\hline & Sem & .90 & 0.99 & 1.35 & 2.06 & 2.60 \\
\hline & $\mathrm{CD}$ at $5 \%$ & 2.63 & 2.90 & 3.96 & 6.01 & 7.60 \\
\hline
\end{tabular}

Table.5 Effect of different treatments on weed control efficiency and weed index

\begin{tabular}{|c|l|c|c|}
\hline $\begin{array}{c}\mathbf{T r} \\
\text { No. }\end{array}$ & Treatments & $\begin{array}{c}\text { Weed } \\
\text { index (\%) }\end{array}$ & $\begin{array}{c}\text { WCE (\%) } \\
\text { at harvest }\end{array}$ \\
\hline $\mathbf{T}_{\mathbf{1}}$ & Alachlor 50 EC, 2.0 kg/ha (Pre.eme.) & 8.3 & 52.29 \\
\hline $\mathbf{T}_{\mathbf{2}}$ & Pendimethalin 30 EC, 750 g/ha (pre.eme) & 10.26 & 55.87 \\
\hline $\mathbf{T}_{\mathbf{3}}$ & Chlorimuron-ethyl 25\% WP, 9.37 g/ha, post eme. (20 DAS) & 6.91 & 75.14 \\
\hline $\mathbf{T}_{\mathbf{4}}$ & Fenoxaprop-p-ethyl 9\% EC,67.5 g/ha post eme.(20 DAS) & 2.3 & 97.98 \\
\hline $\mathbf{T}_{\mathbf{5}}$ & $\begin{array}{l}\text { Chlorimuron ethyl + Fenoxaprop-ethyl, 9.37 g/ha + 67.5 } \\
\text { g/ha, post eme. }\end{array}$ & 4.95 & 97.84 \\
\hline $\mathbf{T}_{\mathbf{6}}$ & Quizalafop-p-ethyl 5\% EC, 50 g/ha, post eme. (15 DAS) & 10.26 & 41.93 \\
\hline $\mathbf{T}_{\mathbf{7}}$ & Imazethapyr 5\% EC, 75 g/ha, post eme. (25 DAS) & 13.61 & 46.15 \\
\hline $\mathbf{T}_{\mathbf{8}}$ & Control (unweeded) & 36.22 & 0.00 \\
\hline $\mathbf{T}_{\mathbf{9}}$ & Weed free & 0.00 & 98.25 \\
\hline
\end{tabular}


Table.6 Plant population of soybean as affected by different treatments

\begin{tabular}{|c|l|c|c|}
\hline $\begin{array}{c}\text { Tr. } \\
\text { No }\end{array}$ & Treatments & \multicolumn{2}{|c|}{$\begin{array}{c}\text { Plant population/ } \\
\text { running meter }\end{array}$} \\
\cline { 2 - 4 } & & 20 DAS & At harvest \\
\hline $\mathbf{T}_{\mathbf{1}}$ & Alachlor 50 EC, 2.0 kg/ha (Pre.eme.) & 16.40 & 16.10 \\
\hline $\mathbf{T}_{\mathbf{2}}$ & Pendimethalin 30 EC, 750 g/ha (Pre.eme.) & 16.20 & 16.10 \\
\hline $\mathbf{T}_{\mathbf{3}}$ & Chlorimuron-ethyl 25\% WP, 9.37 g/ha, post eme. (20 DAS) & 16.30 & 16.40 \\
\hline $\mathbf{T}_{\mathbf{4}}$ & Fenoxaprop-p-ethyl 9 \% EC, 67.5 g/ha post eme.(20 DAS) & 16.10 & 16.50 \\
\hline $\mathbf{T}_{\mathbf{5}}$ & \begin{tabular}{l} 
Chlorimuron ethyl + Fenoxaprop-ethyl, 9.37 g/ha + 67.5 g/ha, \\
\hline
\end{tabular} & 16.30 & 16.10 \\
\hline $\mathbf{T}_{\mathbf{6}}$ & Qust eme. & 16.50 & 16.10 \\
\hline $\mathbf{T}_{\mathbf{7}}$ & Imazethapyr 5 \% EC, 50 g/ha, post eme. (25 DAS) & 16.50 & 15.10 \\
\hline $\mathbf{T}_{\mathbf{8}}$ & Control (unweeded) & 15.30 & 15.80 \\
\hline $\mathbf{T}_{\mathbf{9}}$ & Weed free & 16.40 & 17.00 \\
\hline & SEm & 1.30 & 1.35 \\
\hline & CD at 5\% & $\mathrm{NS}$ & $\mathrm{NS}$ \\
\hline
\end{tabular}

Table.7 Number of trifoliate leaves and root nodules/plant as affected by different treatments

\begin{tabular}{|c|c|c|c|c|c|c|}
\hline \multirow[t]{2}{*}{$\begin{array}{l}\text { Tr. } \\
\text { No }\end{array}$} & \multirow[t]{2}{*}{ Treatments } & \multicolumn{4}{|c|}{$\begin{array}{c}\text { Average number of trifoliate } \\
\text { leaves/plant }\end{array}$} & \multirow{2}{*}{$\begin{array}{c}\text { No. of } \\
\text { nodules/plan } \\
t\end{array}$} \\
\hline & & $\begin{array}{c}30 \\
\text { DAS }\end{array}$ & $\begin{array}{c}\text { 60 } \\
\text { DAS }\end{array}$ & $\begin{array}{c}75 \\
\text { DAS }\end{array}$ & $\begin{array}{c}\text { At } \\
\text { harvest }\end{array}$ & \\
\hline $\mathbf{T}_{1}$ & Alachlor 50 EC, 2.0 kg/ha (Pre.eme.) & 7.61 & 22.40 & 20.20 & 15.70 & 25.00 \\
\hline $\mathbf{T}_{2}$ & Pendimethalin 30 EC, $750 \mathrm{~g} / \mathrm{ha}$ (Pre.eme.) & 7.89 & 21.60 & 19.40 & 14.90 & 24.90 \\
\hline $\mathbf{T}_{\mathbf{3}}$ & $\begin{array}{l}\text { Chlorimuron-ethyl } 25 \% \text { WP, } 9.37 \mathrm{~g} / \mathrm{ha} \text {, post } \\
\text { eme. (20 DAS) }\end{array}$ & 8.80 & 23.90 & 22.60 & 17.10 & 26.40 \\
\hline $\mathbf{T}_{4}$ & $\begin{array}{l}\text { Fenoxaprop-p-ethyl } 9 \% \text { EC, } 67.5 \text { g/ha post } \\
\text { eme.(20 DAS) }\end{array}$ & 9.19 & 26.40 & 24.20 & 20.20 & 28.80 \\
\hline $\mathbf{T}_{\mathbf{5}}$ & $\begin{array}{l}\text { Chlorimuron ethyl + Fenoxaprop-ethyl, } 9.37 \\
\text { g/ha + } 67.5 \mathrm{~g} / \mathrm{ha} \text {, post eme. }\end{array}$ & 9.10 & 24.40 & 21.10 & 17.50 & 26.30 \\
\hline $\mathbf{T}_{6}$ & $\begin{array}{l}\text { Quizalafop-p-ethyl } 5 \% \text { EC, } 50 \mathrm{~g} / \mathrm{ha} \text {, post } \\
\text { eme. (15 DAS) }\end{array}$ & 7.69 & 21.00 & 18.80 & 16.70 & 24.40 \\
\hline $\mathbf{T}_{7}$ & $\begin{array}{l}\text { Imazethapyr } 5 \% \mathrm{EC}, 50 \mathrm{~g} / \mathrm{ha} \text {, post eme. ( } 25 \\
\text { DAS) }\end{array}$ & 7.58 & 21.10 & 18.10 & 14.60 & 21.60 \\
\hline $\mathbf{T}_{8}$ & Control (unweeded) & 6.15 & 19.30 & 17.30 & 15.65 & 21.50 \\
\hline \multirow[t]{3}{*}{$\mathbf{T}_{9}$} & Weed free & 9.58 & 27.40 & 25.50 & 21.60 & 30.70 \\
\hline & SEm & 0.47 & 1.09 & 1.15 & 1.26 & 1.12 \\
\hline & $\mathrm{CD}$ at $5 \%$ & 1.39 & 3.20 & 3.36 & 3.69 & 3.27 \\
\hline
\end{tabular}


Table.8 Seed yield and stover yield (g/plant) as influenced by different treatments

\begin{tabular}{|c|l|c|c|}
\hline $\begin{array}{c}\text { Tr. } \\
\text { No }\end{array}$ & Treatments & $\begin{array}{c}\text { Seed } \\
\text { yield/ } \\
\text { plant }(\mathbf{g})\end{array}$ & $\begin{array}{c}\text { Stover } \\
\text { yield/ } \\
\text { plant (g) }\end{array}$ \\
\hline $\mathbf{T}_{\mathbf{1}}$ & Alachlor 50 EC, 2.0 kg/ha (Pre.eme.) & 4.38 & 6.05 \\
\hline $\mathbf{T}_{\mathbf{2}}$ & Pendimethalin 30 EC, 750 g/ha (Pre.eme.) & 4.25 & 5.90 \\
\hline $\mathbf{T}_{\mathbf{3}}$ & Chlorimuron-ethyl 25\% WP, 9.37 g/ha, post eme. (20 DAS) & 4.79 & 6.75 \\
\hline $\mathbf{T}_{\mathbf{4}}$ & Fenoxaprop-p-ethyl 9\% EC, 67.5 g/ha post eme. (20 DAS) & 5.20 & 7.68 \\
\hline $\mathbf{T}_{\mathbf{5}}$ & Chlorimuron ethyl + Fenoxaprop-ethyl, 9.37 g/ha + 67.5 & 4.88 & 7.12 \\
\hline & g/ha, post eme. & & \\
\hline $\mathbf{T}_{\mathbf{6}}$ & Quizalafop-p-ethyl 5\% EC, 50 g/ha, post eme. (15 DAS) & 4.10 & 5.85 \\
\hline $\mathbf{T}_{\mathbf{7}}$ & Imazswthapyr 5\% EC, 50 g/ha, post eme. (25 DAS) & 4.00 & 5.15 \\
\hline $\mathbf{T}_{\mathbf{8}}$ & Control (unweeded) & 3.78 & 4.82 \\
\hline $\mathbf{T}_{\mathbf{9}}$ & Weed free & 5.32 & 8.10 \\
\hline & Sem & 0.23 & 0.65 \\
\hline & CD at 5\% & 0.69 & 1.88 \\
\hline
\end{tabular}

Table.9 Mean biological yield, grain yield and stover yield (t/ha) as influenced by different treatments

\begin{tabular}{|c|l|c|c|c|}
\hline $\begin{array}{c}\text { Tr. } \\
\text { No. }\end{array}$ & Treatments & $\begin{array}{c}\text { Biological } \\
\text { yield (t/ha) }\end{array}$ & \multicolumn{2}{|c|}{ Yield (t/ha) } \\
\hline $\mathbf{T}_{\mathbf{1}}$ & Alachlor 50 EC, 2.0 kg/ha (Pre.eme.) & 2.72 & 1.31 & 1.41 \\
\hline $\mathbf{T}_{\mathbf{2}}$ & Pendimethalin 30 EC, 750 g/ha (Pre.eme.) & 2.70 & 1.29 & 1.42 \\
\hline $\mathbf{T}_{\mathbf{3}}$ & Chlorimuron-ethyl 25\% WP, 9.37 g/ha, post eme. (20 DAS) & 2.73 & 1.33 & 1.40 \\
\hline $\mathbf{T}_{\mathbf{4}}$ & Fenoxaprop-p-ethyl 9\% EC, 67.5 g/ha post eme. (20 DAS) & 2.87 & 1.40 & 1.47 \\
\hline $\mathbf{T}_{\mathbf{5}}$ & Chlorimuron ethyl + Fenoxaprop-ethyl, 9.37 g/ha +67.5 & 2.73 & 1.36 & 1.37 \\
& g/ha, post eme. & & & \\
\hline $\mathbf{T}_{\mathbf{6}}$ & Quizalafop-p-ethyl 5\% EC, 50 g/ha, post eme. (15 DAS) & 2.74 & 1.29 & 1.46 \\
\hline $\mathbf{T}_{\mathbf{7}}$ & Imazethapyr 5\% EC, 50 g/ha, post eme. (25 DAS) & 2.63 & 1.24 & 1.39 \\
\hline $\mathbf{T}_{\mathbf{8}}$ & Control (unweeded) & 2.05 & 0.91 & 1.14 \\
\hline $\mathbf{T}_{\mathbf{9}}$ & Weed free & 2.99 & 1.43 & 1.55 \\
\hline & SEm & 0.08 & 0.05 & 0.06 \\
\hline & CD at 5\% & 0.23 & 0.15 & 0.17 \\
\hline
\end{tabular}




\section{Number of root nodules per plant}

The number of root nodules per plant was highest (30.70/plant) in treatment $\mathrm{T}_{9}$-Weed free plot followed by $\mathrm{T}_{4}$, fenoxaprop-p-ethyl $9 \%$ EC (28.80/plant) and significantly superior to $\mathrm{T}_{8}$ (control) in which 21.50 number of root nodules/plant were recorded (Table 7).

\section{Studies on yield parameters}

\section{Seed and Stover yield/plant}

The perusal of data revealed that the treatment $\mathrm{T}_{9}$-weed free plot registered highest seed yield/plant $(5.32 \mathrm{~g})$ followed by treatment $\mathrm{T}_{4-}$ fenoxaprop-p-ethyl in which $5.20 \mathrm{~g}$ seed yield/plant was recorded (Table 8 ).

In case of stover yield ( $\mathrm{g}$ ) per plant also, the highest value of $8.10 \mathrm{~g} /$ plant was recorded under treatment $\mathrm{T}_{4}$, fenoxaprop-p-ethyl. While the lowest stover yield of $4.82 \mathrm{~g} / \mathrm{plant}$ was recorded under $T_{8}$, control. There was no significant difference among the treatments of chemical herbicides but all the treatments proved superior over control.

\section{Biological yield (t/ha)}

Blological yield (t/ha), which comprised of the yield of stover ( $t / h a)$ plus the grain of seed yield ( $\mathrm{t} / \mathrm{ha}$ ) has been given in Table 9 along with seed and stover yield (t/ha) separately also to assess the influence of the herbicidal treatments as treatments of control.

\section{Grain yield (t/ha)}

The highest grain yield of $1.43 \mathrm{t} / \mathrm{ha}$ was recorded under treatment $\mathrm{T}_{9}$-weed free plot followed by $1.4 \mathrm{t} / \mathrm{ha}$ under treatment $\mathrm{T}_{4-}$ fenoxaprop-p-ethyl 9\% EC while the lowest grain yield of 0.91 t/ha was obtained under treatment $\mathrm{T}_{8}$ (control). Treatment $\mathrm{T}_{3}$ and $\mathrm{T}_{5}$ with grain yield of 1.33 and 36 tonne per hectare were recorded at par while treatment $\mathrm{T}_{2}$ and $\mathrm{T}_{6}$ both with grain yield of $1.29 \mathrm{t} / \mathrm{ha}$ were found exactly at par with each other.

\section{Stover yield (kg/ha)}

The highest amount of stover yield (1.55 t/ha) of was recorded under $\mathrm{T}_{9}$-weed free plot followed by $1.47 \mathrm{t} / \mathrm{ha}$ under treatment $\mathrm{T}_{4}$ while the lowest stover yield of $1.14 \mathrm{t} / \mathrm{ha}$ was recorded under $\mathrm{T}_{8}$-weed check.

\section{References}

Anonymous (2014). Soybean Processors Association of India, SOPA Souvenir. All India Conversation of Oilseed and Oil Trade and Industries, 2014, p.15.

Bhan, M. and Kewat, M.L. (2003). Activity and persistence of pendimethalin applied pre- emergence to soybean in Vertisol, Ann. Agri. Res., New Series, 24(4): 978-982.

Halvankar,G.B., Varghese, P., Taware, S.P. and Raut, V.M. (2005). Effect of herbicide on weed dynamics and yield of soybean (Glycine max (L) Merrill). $J$. Maha. Agril. Univ; 30(1): 35-37.

Chetan F, Cornel C, Rusu T and Simon A. (2015). Determining influence on the cultivation technology on weeds and soybean production. Production Environment.; 8, 211 - 215.

Dwivedi, R.K., Pandre, N.K. and Ahirwar, M.K. (2019). Integrated Weed Management in Soybean through Front Line Demonstration in Farmer's Field. International Journal of Current Microbiology and Applied Sciences; 8(11): 880-883

Kheriya A, Jha A. K. and Dubey J. (2016). Effect of Chemical Weed Control on Weed Flora and Yield of Soybean; Advances in Life Sciences 5(16).

Kulal D.A, Dhaigude G.S. and Adat S.S., 
(2017). Evaluation of efficacy of post emergence herbicides for weed control in soybean under Marathwada region. International Journal of Agricultural Sciences; 13(1): 53-55

Patel, A., Spare, N. and Malgaya, G. (2019). Bio-Efficacy of Post Emergence Herbicides against Weed Control in Soybean. International Journal of Current Microbiology and Applied Sciences.; 8(4): 1964-1974

Patil, A.S., Bhavsar, M.S., Deore, P.S. and Raut, D.M. (2018). Effect of Integrated Weed Management on Weed Dynamics of Soybean [Glycine max (L.) Merill] under Junagadh, India. International Journal of Current Microbiology and Applied Sciences; 7(1): 1110-1115.

Prachand S, Kubde KJ and Bankar S. (2014). Effect of chemical weed control on weed parameters, growth, yield attributes, yield and economics in Soybean (Glycine max). American Eurasian Journal Agricultural and Environment Science.; 14 (8): 698-701.

Sandil M.K., Sharma J.K., Sanodiya, P. and Pandey, A. (2015). Bio-efficacy on tank mixed Propaquizafop and Imazethapyr against weeds in soybean. Indian Journal of Weed Science.; 47(2): 158162.

Sharma, G.D., Sharma, J.J. and Sonisood (2004). Evaluation of alachlor, metachlor and pendimethanlin for weed control in Rajmash (Phaseolus valgaris L.) in cold desert of North Western Himalayas. Indian J. Weed Sci., 36(3\&4): 287-289.

Thakre, S.S., Deshmukh, J.P., Shingrup, P.V., Pawar, P.M. and Ghlop, A.N. (2015). Efficacy of different new herbicides against weed flora in soybean (Glycine max (L.) Merrill). Plant Archives; 15 (1): 217-220.

Tiwari, J.P., Kurchania, S.P., Paradkar, N.R. and Bhalla, S.P. (1997). Varietal susceptibility and weed control efficiency of fluazifop-p-butyl in soybean (Glycine max). Indian J. agric. Sci., 67(4): 147-149.

Vyas, M.D., Singh, S. and Singh, P.P. (2000). Weed management in soybean (Glycine max (L.) Merrill). Annals of Plant Protection Sci., 8(1): 76-78.

\section{How to cite this article:}

Gabu Singh Gathiye, Subhash Bhanwar and Vishal Verma. 2020. Influence of Pre and Postemergence Herbicides on Weed Dynamics, Growth and Yield of Soybean (Glycine max L.). Int.J.Curr.Microbiol.App.Sci. 9(05): 2975-2986. doi: https://doi.org/10.20546/ijcmas.2020.905.353 